\title{
Tecnologias móveis para auxiliar no manejo terapêutico de leishmaniose tegumentar: uma revisão integrativa
}

Mobile technologies to aid in the therapeutic management of tegumentary leishmaniasis: an integrative review

Tecnologías móviles para ayudar en el manejo terapéutico de la leishmaniasis tegumentaria: una revisión integrativa

\section{Resumo}

Objetivo: demostrar como a utilização de recursos tecnológicos móveis pode auxiliar no atendimento à pacientes com leishmaniose tegumentar (LT). Métodos: trata-se de uma revisão integrativa com abordagem qualitativa, em que foi realizado um levantamento bibliográfico das publicações realizadas entre 2017 e 2021, nas bases de dados LILACS, MEDLINE e SciELO, bem como na biblioteca virtual Google Scholar. Resultados: a amostra final desta revisão foi constituída por oito publicações, sendo quatro delas realizadas no Brasil. Alguns estudos apresentaram o desenvolvimento de aplicativos móveis como o LeishCare ${ }^{\circledR}$ e o Leish Heal, enquanto outros abordaram a utilização de sistemas de softwares que possibilitam o acompanhamento das lesões dos pacientes ao longo do tratamento através de fotografias. A criação de bancos de dados para assuntos dermatológicos e a utilização de ferramentas comuns de comunicação já existentes em smartphones conectados à internet, tal como aplicativos de trocas de mensagens que permitam o envio de imagens, e-mail, redes sociais também demonstraram como esses recursos tecnológicos podem auxiliar na assistência à pacientes com LT. Conclusão: observa-se a importância do novel de investigações nessa área, principalmente no Brasil e em regiões consideradas endêmicas, visando o acompanhamento, tratamento e melhoria na qualidade da assistência e da terapêutica da leishmaniose.

Palavras-chave: Atenção à saúde; Leishmaniose cutânea; Revisão sistemática; Aplicativos móveis.

\begin{abstract}
Objective: demonstrate how the use of mobile technological resources can help in the care of patients with tegumentary leishmaniasis (TL). Methods: this is an integrative review with a qualitative approach, with a bibliographic survey of publications carried out between 2017 and 2021 was carried out, in the LILACS, MEDLINE and SciELO databases, as well as in the Google Scholar virtual library. Results: the final sample of this review consisted of eight publications, four of which were carried out in Brazil. Some studies showed the development of mobile applications such as LeishCare ${ }^{\circledR}$ and Leish Heal, while others addressed the use of software systems that
\end{abstract}


enable the monitoring of patients' injuries throughout treatment through photographs. The creation of databases for dermatological subjects and the use of common communication tools that already exist on smartphones connected to the internet, such as messaging apps that allow the sending of images, email, social networks also demonstrated how these features technological devices can help in the care of patients with TL. Conclusion: the importance of new investigations in this area is observed, mainly in Brazil and in regions considered endemic, aiming at monitoring, treating and improving the quality of care and treatment of leishmaniasis.

Keywords: Delivery of Health Care; Leishmaniasis, cutaneous; Systematic review; Mobile applications.

\begin{abstract}
Resumen
Objetivo: demostrar cómo el uso de recursos tecnológicos móviles puede ayudar en la atención de pacientes con leishmaniasis tegumentaria (LT). Métodos: se trata de una revisión integradora con enfoque cualitativo, donde se realizó un relevamiento bibliográfico de publicaciones realizadas entre 2017 y 2021, buscado en las bases de datos LILACS, MEDLINE y SciELO, así como en la biblioteca virtual Google Scholar. Resultados: la muestra final de esta revisión estuvo constituida por ocho publicaciones, cuatro de ellas realizadas en Brasil. Algunos estudios han mostrado el desarrollo de aplicaciones móviles como LeishCare ${ }^{\circledR}$ y Leish Heal, mientras que otros han abordado el uso de sistemas de software que permiten monitorear las lesiones de los pacientes durante el tratamiento a través de fotografías. La creación de bases de datos para temas dermatológicos y el uso de herramientas de comunicación comunes que ya existen en los teléfonos inteligentes conectados a Internet, como las aplicaciones de mensajería que permiten el envío de imágenes, correo electrónico, redes sociales, también demostraron cómo estas características de los dispositivos tecnológicos pueden ayudar en la atención de pacientes con LT. Conclusión: se observa la importancia de nuevas investigaciones en esta área, principalmente en Brasil y en regiones consideradas endémicas, con el objetivo de monitorear, tratar y mejorar la calidad de la atención y el tratamiento de la leishmaniasis.
\end{abstract}

Palabras clave: Atención a la Salud; Leishmaniasis cutánea; Revisión sistemática; Aplicaciones móviles.

\title{
1. Introdução
}

As Leishmanioses são um importante problema de saúde pública nas Américas devido a sua ampla distribuição e elevada prevalência (Lainson \& Shaw, 1987). Seu complexo ciclo de transmissão compreende diferentes espécies de parasitos, reservatórios e vetores (Morais, 2015). São doenças infecciosas, não contagiosas, causadas por protozoários de gênero Leishmania, com várias espécies envolvidas e apresentando as formas clínicas de Leishmaniose Tegumentar (LT), podendo ser cutânea e mucosa, e Leishmaniose visceral (LV) (Pace, 2014; Gontijo et al., 2003).

Segundo a Organização Pan-americana da Saúde OPAS/OMS (Informe de Leishmanioses nº 8), 17 dos 18 países das Américas são endêmicos para leishmanioses (Opas/Oms, 2019). Entre os anos de 2001 a 2019 foram notificados 1.028 .054 casos de Leishmaniose Cutânea (LC) e Mucosa (LM) pelos países endêmicos das Américas (com exceção da Guiana Francesa, uma vez que país reporta seus dados diretamente para a França), com uma média de 54.108 casos por ano (Opas/Oms, 2020). Em 2019, o Brasil esteve entre os países com o maior número de casos de leishmanioses confirmados (Opas/Oms, 2020).

A Leishmaniose está incluída entre as doenças tropicais negligenciadas (DNTs), as quais acometem populações em vulnerabilidade, em especial onde não se dispõe de saneamento básico adequado e que estão sujeitas ao contato direto com vetores e reservatórios de agentes infecciosos e parasitários (Who, 2017).

As doenças são classificadas como "negligenciadas" devido à falta de interesse do poder público e também das grandes indústrias em desenvolver produtos e tecnologias para o tratamento, prevenção e controle dessas doenças (MartinsMelo et al., 2016; Hotez et al., 2006; Who, 2010).

Ao todo existem cerca de 20 doenças negligenciadas listadas pela Organização Mundial da Saúde (OMS/WHO), onde, além da Leishmaniose, tem-se: Úlcera de Buruli; Doença de Chagas; Dengue e Chikungunya; Dracunculíase (doença do verme-da-Guiné); Equinococose; Trematodíases de origem alimentar; Tripanossomíase africana (doença do sono); Hanseníase; Filariose Linfática; Micetoma, cromoblastomicose e outras micoses profundas; Oncocercose; Raiva; Escabiose e outras ectoparasitoses; Esquistossomose; Helmintíases transmitidas pelo solo; envenenamento por picada de cobra; Teníase e Cisticercose; Tracoma; Bouba (Who, 2020).

$\mathrm{Na}$ LT, as infecções na forma cutânea, são caracterizadas por úlcera, geralmente indolor que costuma localizar-se em 
áreas expostas da pele; porém quando associada a infecção bacteriana, pode causar dor local e produzir exsudato seropurulento que, ao dessecar-se em crostas, recobre total ou parcialmente o fundo da úlcera (Mota et al., 2011; Brasil, 2017). Já na forma mucosa, a doença é a mais agressiva e expressa-se por lesões destrutivas localizadas nas mucosas das vias aéreas superiores, sendo que a forma clássica da forma mucosa é secundária à lesão cutânea (Mota et al., 2011; Brasil, 2010).

A transmissão da LT está relacionada a atividades nas matas, como extrativismo, abertura de estradas, caça, treinamento militar e atividades de lazer, como trilhas entre outras, acomete mais as pessoas do sexo masculino e em idade produtiva (Pessoa \& Martins, 1982; Falqueto \& Sessa, 2002; Curti et al., 2009; Guimarães, 2010).

De acordo com a OMS, das mortes de pacientes em tratamento de LT, 81,8\% foram em pacientes maiores de 50 anos, o que pode estar associado às possíveis complicações causadas pelo uso dos medicamentos e doenças de base, contudo não há estudos que comprovem tal suposição (Opas/Oms, 2019).

Segundo Souza (2018, p. 80), a mortalidade por LT é um fenômeno recente, cujos "fatores não relacionados diretamente a doença despontam como causa provável do óbito, como por exemplo, fatores socioeconômicos e relacionados aos serviços de saúde". Nesse sentido, tem-se que em locais de menor incidência da doença, é possível se deparar com profissionais com pouca ou nenhuma experiência no manejo clínico de pacientes com LT, acarretando em deslocamentos para outros municípios para dar seguimento ao tratamento.

Através de uma análise espaço-temporal, Souza (2018) evidenciou a associação do óbito com a toxicidade relacionada ao tratamento, e acompanha o desfecho final de cada caso clínico, podendo evoluir para cura da LT, morte por LT, ou morte associadas a outras causas. Essa associação está relacionada, dentre outros fatores, a presença de comorbidades e complicações ao longo do tratamento. Foi observado que a infecção por Leishmania pode não ser a causa direta dos óbitos, cuja causa imediata parece ser infecção bacteriana e disfunção orgânica desencadeada pelo tratamento.

Atualmente, a droga de primeira linha para tratamento da LT, no Brasil, é o antimoniato de meglumina que, apesar de sua toxicidade e das queixas quanto a sua administração, é considerada bastante eficaz (Schubach \& Conceição-Silva, 2014). Embora ainda não seja disponibilizado pelo Ministério da Saúde, outro medicamento existente para o tratamento da LT é a miltefosina (hexadecilfosfocolina), cujo uso é oral. Vale ressaltar que, por ser administrado por via oral, a adesão ao tratamento da doença seria facilitada, uma vez que o medicamento poderia ser dispensado para o paciente logo após o diagnóstico da doença, permitindo ainda que o doente fosse tratado em sua própria residência (Conitec, 2018).

Tão importante quanto oferecer alternativas para o tratamento da LT é a necessidade de enfrentar a grande fragilidade dos dados do sistema de vigilância do Brasil, como falhas de preenchimento, diagnósticos incorretos e evolução notificada de forma incorreta. A falta de um sistema que monitore, registre e comunique, dificulta o bom monitoramento do paciente e o desfecho da doença (Souza, 2018).

Observa-se a necessidade de um sistema para unificar e gerir esses dados sobre a doença em seus principais aspectos. Assim, o desenvolvimento de ferramentas tecnológicas acessíveis, tais como aplicativos móveis tem se mostrado uma excelente alternativa do enfrentamento dessa problemática, auxiliando os profissionais no manejo dos pacientes com LT.

Portanto, a presente investigação é uma revisão integrativa da literatura que teve como objetivo demonstrar como a utilização de recursos tecnológicos móveis pode auxiliar no atendimento às pessoas acometidas com LT.

\section{Metodologia}

Visando reunir evidências e dados que acrescentem informações ou debatam sobre a temática deste estudo, "a revisão integrativa oferece aos profissionais de diversas áreas de atuação na saúde o acesso rápido aos resultados relevantes de pesquisas que fundamentam as condutas ou a tomada de decisão, proporcionando um saber crítico" (Mendes et al., 2008, p. 765). 
A consulta dos estudos foi efetuada por acesso on-line em abril de 2021. A seleção dos artigos foi realizada nas bases de dados ScIELO (Scientifie Eletronic Library Online), LILACS (Literatura Latino-Americana e do Caribe em Ciências da Saúde), Medline e PubMed, bem como na biblioteca eletrônica Google Scholar (Google Acadêmico).

Os descritores utilizados nas buscas foram estabelecidos de acordo com o DeCS (Descritores em Ciências de Saúde), sendo eles: Leishmaniose Tegumentar Americana (Leishmaniasis Cutaneous / Leishmaniasis Cutánea); Tecnologias em Saúde (Biomedical Technology / Tecnología Biomédica); Tecnologia e Aplicativos de Software (Technology / Tecnología); e Dispositivos móveis (Mobile Devices / Dispositivos Móviles). Os termos foram cruzados como descritores e também como palavras do título e do resumo.

Como critérios de inclusão foram considerados os artigos publicados entre janeiro de 2017 e janeiro de 2021 tanto em português, inglês e espanhol, que possuíam no mínimo dois dos descritores utilizados para terem seus resumos lidos. Aqueles que estavam com duplicações ou não apresentavam o resumo foram excluídos das análises.

Assim, após a leitura dos resumos, foram eleitos os estudos com potencial para a leitura na íntegra, os quais atendiam as expectativas desta pesquisa. Ao final, foram incluídos nesta revisão integrativa as publicações que de fato versaram sobre como a utilização de recursos tecnológicos móveis no atendimento à pacientes com LT.

\section{Resultados}

A amostra final desta revisão foi constituída por 8 artigos científicos, selecionados para a leitura analítica de acordo com os critérios de inclusão previamente estabelecidos (Figura 1). 
Figura 1 - Fluxograma dos processos de busca, seleção, exclusão e inclusão das publicações para a revisão integrativa.

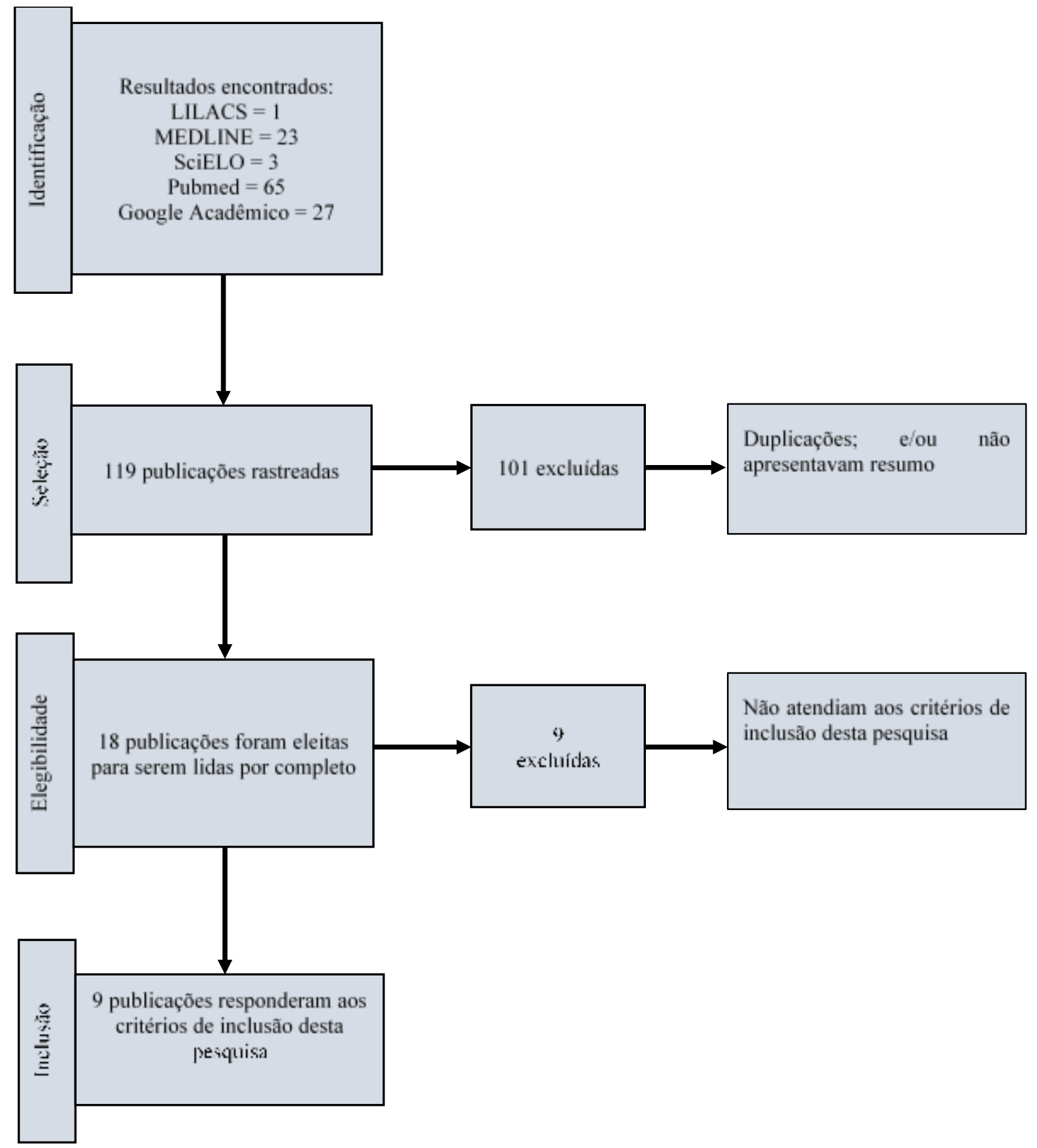

Fonte: Autores (2021).

A Tabela 1 apresenta os cinco estudos selecionados para esta revisão integrativa, com seus respectivos autores e ano de publicação, títulos, local de busca e suas principais conclusões. Somente foram selecionadas as publicações encontradas nas bases de dados MEDLINE e PubMed, e na biblioteca digital Google Scholar (Tabela 1). Por sua vez, LILACS e SciELO não apresentaram resultados que abrangessem os critérios de inclusão ao se realizar as buscas a partir do cruzamento dos descritores. 
Tabela 1 - Estudos publicados entre 2017 e 2021 nas bases de dados pesquisadas que foram selecionados para a revisão integrativa.

\begin{tabular}{|c|c|c|c|}
\hline $\begin{array}{l}\text { Autor(es) e ano } \\
\text { de publicação }\end{array}$ & $\begin{array}{l}\text { Título } \\
\text { da pesquisa }\end{array}$ & $\begin{array}{l}\text { Local } \\
\text { de busca }\end{array}$ & Principais conclusões \\
\hline $\begin{array}{l}\text { Rubiano et al. } \\
\text { (2021) }\end{array}$ & $\begin{array}{l}\text { Adaptation and performance of a } \\
\text { mobile application for early detection } \\
\text { of cutaneous leishmaniasis }\end{array}$ & Medline & $\begin{array}{l}\text { A tradução de uma regra de predição validada para uma } \\
\text { tecnologia de saúde móvel mostrou o potencial de } \\
\text { melhorar a capacidade dos agentes comunitários de saúde } \\
\text { e profissionais da saúde para fornecer atendimento } \\
\text { oportuno e acesso a serviços de saúde para populações } \\
\text { carentes. Essas descobertas apoiam o uso de ferramentas } \\
\text { de saúde móvel para pesquisa e saúde em DTN. }\end{array}$ \\
\hline $\begin{array}{l}\text { Silva et al. } \\
(2020)\end{array}$ & $\begin{array}{l}\text { LeishCare®: A Software Designed for } \\
\text { the Management of Individuals with } \\
\text { Leishmaniases }\end{array}$ & PubMed & $\begin{array}{l}\text { O LeishCare }{ }^{\circledR} \text { foi bem aceito em áreas endêmicas e pode } \\
\text { ser utilizado por profissionais de saúde em sua prática } \\
\text { diária. O aplicativo traz benefícios para seus usuários } \\
\text { mesmo quando aplicado em pequena escala. }\end{array}$ \\
\hline $\begin{array}{l}\text { Devrim et al. } \\
(2019)\end{array}$ & $\begin{array}{l}\text { Reliability and accuracy of } \\
\text { smartphones for } \\
\text { Paediatric infectious disease } \\
\text { consultations for children with rash in } \\
\text { the paediatric } \\
\text { emergency department }\end{array}$ & PubMed & $\begin{array}{l}\text { O diagnóstico inicial, que foi realizado via WhatsApp em } \\
\text { um smartphone, foi idêntico ao diagnóstico final em } \\
96,3 \% \text { dos casos. Os diagnósticos iniciais incompatíveis } \\
\text { incluíram } 4 \text { casos de sarampo, } 1 \text { síndrome da pele } \\
\text { escaldada estafilocócica caso, } 1 \text { caso de leishmaniose } \\
\text { cutânea e } 1 \text { caso de erupção cutânea petequial. }\end{array}$ \\
\hline Ilha (2019) & $\begin{array}{l}\text { Elaboração de banco de dados para } \\
\text { desenvolvimento de Aplicativo móvel } \\
\text { direcionado ao aprendizado de } \\
\text { dermatologia }\end{array}$ & $\begin{array}{l}\text { Google } \\
\text { Scholar }\end{array}$ & $\begin{array}{l}\text { A leishmaniose tegumentar teve ênfase por ser abordada } \\
\text { em aproximadamente } 30 \% \text { dos artigos de periódicos } \\
\text { selecionados. [...] A criação de ferramentas de facilitem o } \\
\text { acesso a esse banco de dados, como por exemplo, } \\
\text { aplicativos móveis para celulares, podem viabilizar o fácil } \\
\text { acesso ao conteúdo desenvolvido. }\end{array}$ \\
\hline $\begin{array}{l}\text { Fonseca-Junior } \\
(2018)\end{array}$ & $\begin{array}{l}\text { Desenvolvimento de um software para } \\
\text { dispositivos móveis visando auxiliar no } \\
\text { manejo de indivíduos doentes, } \\
\text { utilizando imagens digitais para estudo } \\
\text { de sua evolução }\end{array}$ & $\begin{array}{l}\text { Google } \\
\text { Scholar }\end{array}$ & $\begin{array}{l}\text { Do ponto de vista de usabilidade a expectativa é que o } \\
\text { aplicativo execute de forma fluida devido a otimização de } \\
\text { recursos. [...] Sua utilização está sendo focada nas áreas } \\
\text { endêmicas de leishmaniose do interior de Minas, e após a } \\
\text { conclusão do projeto será possível avaliar e extrair os } \\
\text { resultados do seu uso. }\end{array}$ \\
\hline $\begin{array}{l}\text { Navarro et al. } \\
(2018)\end{array}$ & $\begin{array}{l}\text { Developing mobile health applications } \\
\text { for neglected tropical disease research }\end{array}$ & Medline & $\begin{array}{l}\text { Combinar o desenvolvimento de tecnologia ágil } \\
\text { (interações rápidas) e amplo compromisso das partes } \\
\text { interessadas, especialmente das comunidades locais, pode } \\
\text { melhorar o desenvolvimento de intervenções, otimizar as } \\
\text { evidências coletadas e acelerar a tradução do } \\
\text { conhecimento em ação. }\end{array}$ \\
\hline Albiero (2017) & $\begin{array}{l}\text { Uma abordagem de teste para } \\
\text { aplicativos Android utilizando os } \\
\text { cenários do Behavior Driven } \\
\text { Development }\end{array}$ & $\begin{array}{l}\text { Google } \\
\text { Scholar }\end{array}$ & $\begin{array}{l}\text { A abordagem proposta foi utilizada em quatro aplicativos } \\
\text { Android e se mostrou útil, uma vez que, em três estudos } \\
\text { de caso foram detectados bugs oriundos de } \\
\text { inconsistências lógicas nos cenários ou elementos não } \\
\text { exercitados pelos cenários. }\end{array}$ \\
\hline $\begin{array}{l}\text { Al-Kamel } \\
(2017)\end{array}$ & $\begin{array}{l}\text { Feasibility of teledermatology in the } \\
\text { eradication of } \\
\text { leishmaniasis from Yemen project }\end{array}$ & $\begin{array}{l}\text { Google } \\
\text { Scholar }\end{array}$ & $\begin{array}{l}\text { O desenvolvimento do programa de Teledermatologia [...] } \\
\text { melhorou vidas e salvou custos de transporte } \\
\text { desnecessários de pacientes com leishmaniose em áreas } \\
\text { rurais desfavorecidas, melhorou a documentação dos } \\
\text { pacientes, aumentou a educação remota e a } \\
\text { conscientização da comunidade sobre a doença, melhorou } \\
\text { o conhecimento da equipe e aumentou as capacidades de } \\
\text { pesquisa da leishmaniose no Iêmen. }\end{array}$ \\
\hline $\begin{array}{l}\text { Taheri et al. } \\
\text { (2017) }\end{array}$ & $\begin{array}{l}\text { Digital volumetric measurement of } \\
\text { cutaneous leishmaniasis lesions: Blur } \\
\text { estimation method }\end{array}$ & PubMed & $\begin{array}{l}\text { O processamento de imagens com a técnica de estimativa } \\
\text { de borrão é um método acurado e preciso para medir o } \\
\text { volume das lesões na leishmaniose tegumentar. }\end{array}$ \\
\hline
\end{tabular}

Fonte: Autores (2021).

\section{Discussão}

De modo geral, para que a confecção e utilização do aplicativo móvel seja viável, alguns aspectos metodológicos devem ser observados. Para Almeida (2017), o software tradicional (ou aplicativo móvel), deve contar com bastante autonomia, ser estruturado e subdividido por funções, além de ser rápido, intuitivo, prático e objetivo.

Trabalhos como o de Rocha et al. (2016), Barra et al. (2017) e Bezerra et al. (2020), voltados para a relação entre 
aplicativos móveis e a saúde, expõem a importância na utilização de aplicativos e softwares de forma ampla na gestão e cuidado em saúde. Para os autores, a procura e oferta dos serviços de saúde sofrerá ou já sofre profundas alterações, que exige um esforço de adaptação, tanto do prestador do serviço em si, quanto para quem atua na área. A utilização dessas tecnologias é um grande auxílio na gestão em saúde, pois melhora a qualidade de vida do usuário, monitora, informa e facilita o acesso a diversos serviços.

Já para de Oliveira e Alencar (2017), o uso de aplicativos móveis também pode ser utilizado como ferramenta para a educação em saúde. Os autores concluíram que aplicativos utilizados na prática educativa aumentam a eficiência de educadores e alunos por automatizar a distribuição, a coleta, a verificação e a documentação de avaliaçães e exames.

Em relação a LT, foi encontrado somente nove estudos envolvendo aplicativos móveis, dentre os quais apenas três foram realizados no Brasil (Silva et al., 2020; Ilha 2019; Fonseca-Júnior 2018). O aplicativo móvel LeishCareß foi desenvolvido no país para o manejo de indivíduos com suspeita de leishmanioses (visceral e tegumentar), voltado para profissionais de saúde em áreas endêmicas, tem como principal objetivo registrar a evolução das lesões cutâneas de maneira remota, além de identificar prováveis riscos de mortes (Silva et al., 2020). O aplicativo mostrou-se promissor para auxiliar os profissionais de saúde nas áreas endêmicas analisadas.

Similarmente, Fonseca-Júnior (2018) desenvolveu um aplicativo para dispositivo móvel direcionado para auxiliar profissionais de saúde no acompanhamento de pacientes com leishmaniose em regiões pobres e com pouca infraestrutura, onde o software foi dimensionado para ser usado em smartphones com poucos recursos para, assim, possibilitar seu uso. Esse aplicativo facilita o acompanhamento das lesões dos pacientes ao longo do tratamento através de fotografias, pois torna possível sincronizar manualmente a foto no momento em que está sendo tirada com uma foto que foi tirada anteriormente. Assim, por exemplo, pode-se acompanhar o processo de cicatrização de uma lesão de LT, sendo possível disponibilizar futuramente um catálogo de imagens sequenciais demonstrando a melhora ou piora do quadro do paciente.

Outro aplicativo desenvolvido em território nacional é o Leish Heal, fruto de uma parceria entre a UFRGS e a Fiocruz-MG (Albiero, 2017). Trata-se de um aplicativo criado com o objetivo de auxiliar profissionais de saúde, médicos e enfermeiros que tratam de pacientes com LT, tendo como funcionalidades o cálculo da área da lesão, cálculo do volume e do limite diário de medicamento que uma pessoa em tratamento pode receber, entre outras. $\mathrm{O}$ aplicativo se encontra em processo de verificação e homologação pela Fiocruz-MG e ainda não está disponível para download.

Os estudos estrangeiros encontrados nesta pesquisa foram desenvolvidos na Colômbia (Rubiano et al., 2021; Navarro et al., 2018), na Turquia (Devrim et al., 2019), no Irã (Taheri et al., 2017) e no Iêmen (Al-Kamel, 2017), onde também apresentaram resultados promissores sobre o tema.

Rubiano et al. (2021) demonstrou um alto nível de concordância bruta entre o aplicativo móvel desenvolvido e o diagnóstico parasitológico de LT. Nesse estudo, o tempo para o diagnóstico foi reduzido significativamente, de 8 para 4 semanas em média, quando os agentes comunitários de saúde conduziram a deteção ativa de casos usando o aplicativo, em comparação com a detecção passiva de casos pelos profissionais da unidade de saúde.

O Guaral/app foi desenvolvido para casos de LT na Colômbia e, diante disso, Navarro et al. (2018) revisaram as principais etapas na criação de tais ferramentas de saúde móvel (mHealth). Os autores observaram que esses recursos devem considerar o contexto sociotécnico usando métodos como análise de sistemas e design centrado no ser humano, baseados no engajamento e interação com todas as partes interessadas. Também enfatizam a usabilidade e descrevem a importância da interdependência do técnico e do humano como considerações para sistemas de saúde móvel em comunidades rurais.

Taheri et al. (2017) projetaram um sistema de software que analisa imagens de lesões de LT a partir de uma técnica fotográfica utilizada juntamente com um algoritmo de processamento de imagem para extrair um mapa tridimensional da lesão a partir de uma imagem bidimensional simples. Esse método recupera a profundidade com base na estimativa de desfoque. Os 
autores concluíram que o processamento de imagens com a técnica de estimativa de borrão é um método acurado e preciso para mensurar o volume das lesões na leishmaniose tegumentar.

Por outro lado, estudos como o de Al-Kamel (2017) e Devrim et al. (2019) não abordaram o desenvolvimento de um aplicativo móvel específico para LT, mas demonstraram que a utilização de ferramentas comuns de comunicação já existentes em smartphones conectados à internet, tal como aplicativos de trocas de mensagens que permitam o envio de imagens, e-mail, redes sociais, sem contar as chamadas telefônicas e SMS, podem ser úteis e preciso o diagnóstico e tratamento em pacientes com leishmaniose.

Diante do exposto, os trabalhos incluídos na revisão integrativa da literatura apresentaram resultados positivos tanto para utilização de aplicativos móveis específicos, como para a utilização de recursos tecnológicos já existentes. Nesse sentido, para auxiliar no desenvolvimento de aplicativos móveis para o diagnóstico e tratamento de pacientes, Ilha (2019) criou um banco de dados dos assuntos dermatológicos mais relevantes, dentre eles a LT. A autora destaca a importância da criação de aplicativos móveis para celular como ferramentas que facilitem o acesso ao conteúdo desenvolvido.

Esses achados são promissores para o acolhimento e manejo terapêutico de LT, uma vez que a certeza do diagnóstico só se obtém através de vários métodos, onde a chance de se encontrar o parasito é inversamente proporcional ao tempo de duração da lesão (Gontijo \& Carvalho, 2003). Segundo a Organização Mundial da Saúde, Who (2016), em apenas 32 dos 88 países afetados pela doença a sua notificação é compulsória; sendo o Brasil um deles (Brasil, 2014).

No Brasil as áreas endêmicas de LT, que antes se concentravam exclusivamente em regiões rurais, agora vem se expandindo também para áreas urbanas. Sendo a Leishmaniose uma enfermidade que avançou para novas regiões do país, muitos profissionais de saúde não estão habituados a reconhecerem a doença. A dificuldade de se chegar a um diagnóstico é grande até mesmo para especialistas em Leishmaniose (Fonseca-Júnior, 2018). A LT pode ter as úlceras confundidas com úlceras traumáticas, úlceras de estase, úlcera tropical, úlceras de membros inferiores por anemia falciforme, piodermites, paracoccidioidomicose entre outras (Brasil, 2005).

Diante disso, compreende-se que o uso de tecnologias que facilitem o diagnóstico e manejo terapêutico da LT é benéfico e deve ser estimulado visando diminuir a incidência e as taxas de mortalidade da doença. Corroborando com Barra et al. (2017), compreende-se que "independentemente do método de desenvolvimento escolhido, as etapas devem ser bem definidas e estruturadas de forma adequada, para que o aplicativo móvel desenvolvido seja útil ao usuário final" (Barra et al., 2017, p. 9).

Ressalta-se também a importância de que, na construção desses aplicativos, sejam considerados os aspectos étnicos de cada região do país, assim, auxiliando os profissionais de saúde no desenvolvimento de práticas cada vez mais abrangentes no cuidado e prevenção da doença.

Nitidamente, os estudos envolvendo a utilização de softwares móveis no acolhimento e tratamento da LT são promissores. Embora o número de publicações sobre o tema seja ainda incipiente, com apenas três estudos desenvolvidos no Brasil entre 2017 e 2021 conforme observado nesta pesquisa, mesmo com algumas limitações, os resultados são positivos.

\section{Conclusão}

Este estudo de revisão integrativa demonstrou como a evolução tecnológica torna possível orientar e alertar os profissionais que realizam o atendimento de pacientes com LT, seja através de ferramentas simples de comunicação ou por aplicativos para dispositivos móveis. Esses recursos têm se apresentado como alternativas viáveis, onde todos os estudos analisados revelaram resultados satisfatórios. Observou-se que esses aplicativos são o que há de mais novo e fácil acessibilidade para os profissionais de saúde, podendo auxiliar na conduta de manejo e segmento do paciente, especialmente em regiões onde os casos de LT são recorrentes. 
É esperado que devido a facilidade, praticidade e acessibilidade, os aplicativos possam auxiliar na identificação de fatores de risco, conduta de pacientes, na elaboração de protocolos de tratamentos, sinalização de hospitais referências, além de informar também as áreas de maior incidência da doença.

Avaliando a proposição desta revisão integrativa frente aos resultados encontrados nos artigos incluídos, observa-se a importância do novel de investigações nessa área, principalmente no Brasil e em regiões consideradas endêmicas, visando o acompanhamento, tratamento e melhoria na qualidade da assistência e da terapêutica da leishmaniose.

Portanto, sugere-se que mais estudos sejam desenvolvidos na área da saúde alinhada ao uso de tecnologias móveis, especialmente para as doenças negligenciadas que acometem populações em vulnerabilidade, sem acesso a saneamento básico adequado e que estão sujeitas ao contato direto com vetores e reservatórios de agentes infecciosos e parasitários, tal como é o caso da Leishmaniose. Espera-se que futuramente os aplicativos móveis façam parte do cotidiano de profissionais da saúde que estejam inseridos em contextos de dificuldades quanto ao atendimento de pacientes.

\section{Referências}

Albiero, F. W. (2017). Uma abordagem de teste para aplicativos Android utilizando os cenários do Behavior Driven Development. [Dissertação de Mestrado, Universidade Federal do Rio Grande do Sul, Instituto de Informática]. http://hdl.handle.net/10183/158399

Al-Kamel, M. A. (2017). Feasibility of teledermatology in the eradication of leishmaniasis from Yemen Project. Glob Dermatol, 4(1), 1-3. https://doi.org/10.15761/GOD.1000206

Almeida, G. A. M. (2017). Fatores de escolha entre metodologias de desenvolvimento de software tradicionais e ágeis. [Dissertação de Mestrado, Escola Politécnica da Universidade de São Paulo]. https://doi.org/10.11606/D.3.2017.tde-11042017-143311

Barra, D. C. C., Paim, S. M. S., Sasso, G. T. M. D., \& Colla, G. W. (2017). Métodos para desenvolvimento de aplicativos móveis em saúde: revisão integrativa da literatura. Texto Contexto Enferm, 26(4), e2260017. http://dx.doi.org/10.1590/0104-07072017002260017

Bezerra, L., Vilhena, B. J., Freitas, R. N., Bastos, Z. R. Z., Teixeira, E., Menezes, E. G., Xavier, C., \& Sicsú, D. A. N. (2020). Aplicativos móveis no cuidado em saúde: uma revisão integrativa. Revista Enfermagem Atual In Derme, $93(31)$, e-020047. https://revistaenfermagematual.com.br/index.php/revista/article/view/760/723

Brasil. (2005). Leishmaniose Tegumentar Americana: Guia de Vigilância Epidemiologica. Secretaria de Vigilância em Saúde, 11(6): 1-30.

Brasil. (2014). Portaria No 1.271, de junho de 2014. Lista Nacional de Notificação Compulsória de doenças, agravos e eventos de saúde pública nos serviços de saúde públicos e privados em todo o território nacional, nos termos do anexo, e dá outras providências. http://bvsms.saude.gov.br/bvs/saudelegis/gm/2014/prt1271_06_06_2014.html

Curti, M. C. M., Silveira, T.G.V., Arraes, S. M. A. A., Bertolini, D. A., Zanzarini, P. D., Venazzi, E. A. S., Fernandes, A. C. S., Teixeira, J. J. V., \& Lonardoni, M. V. C. (2009). Aspectos epidemiológicos da Leishmaniose Tegumentar Americana na Região Nordeste do Estado do Paraná. Rev Ciênc Farm Básica Ap, 30(1), 51-56. http://files.bvs.br/upload/S/1808-4532/2009/v30n1/a005.pdf

Devrim, I., Düzgöl, M., Kara, A., Çağlar, I., Devrim, F., Bayram, N., \& Apa, H. (2019). Reliability and accuracy of smartphones for paediatric infectious disease consultations for children with rash in the paediatric emergency department. BMC Pediatrics, 19(40), 1-5. https://doi.org/10.1186/s12887-019-1416-8

Falqueto, A., \& Sessa, P.A. (2002). Leishmaniose tegumentar americana. In: Veronezi, R. \& Foccacia P. A (org.). Tratado de Infectologia (pp. 1241-53). Editora Atheneu

Fonseca-Junior, G. S. (2018). Desenvolvimento de um software para dispositivos móveis visando auxiliar no manejo de indivíduos doentes, utilizando imagens digitais para estudo de sua evolução. [Dissertação de Mestrado Profissional em Inovação Tecnológica, Universidade Federal do Triângulo Mineiro, Uberaba-MG]. http://bdtd.uftm.edu.br/handle/tede/565

Gontijo, B., \& Carvalho, R. L. M. (2003). Leishmaniose tegumentar americana. Revista da Sociedade Brasileira de Medicina Tropical, 36(1),71-80. https://www.scielo.br/pdf/rsbmt/v36n1/15310.pdf

Guimarães, H. C. Q. C. P. (2010). Leishmaniose - oportunidade para o desenvolvimento de um novo domínio na enfermagem. Acta Paul Enferm., 23(4), vii. https://doi.org/10.1590/S0103-21002010000400001

Hotez, P. J., Molyneux, D. H., Fenwick, A., Ottesen, E., Sachs, S. E., \& Sachs, J. D. (2006). Incorporating a Rapid-Impact Package for Neglected Tropical Diseases with Programs for HIV/AIDS, Tuberculosis, and Malaria. PLoS Medicine, 3(5), e102. https://doi.org/10.1371/journal.pmed.0030102

Ilha, C. S. O. (2019). Elaboração de banco de dados para desenvolvimento de aplicativo móvel direcionado ao aprendizado de dermatologia. [Dissertação de Mestrado, Faculdade de Ciências Médicas da Universidade Estadual de Campinas]. https://doi.org/10.47749/T/UNICAMP.2019.1127294

Lainson, R., \& Shaw, J. J. (1987). Evolution, classification and geographical distribution. In: Peters, W.; Killick-Kendrick, R. (eds.). The Leishmaniasis in Biology and Medicine (pp. 1-120). Academic Press Inc, London. 
Martins-Melo, F. R., Ramos Jr, A. N., Alencar, C. H., \& Heukelbach, J. (2016). Trends and spatial patterns of mortality related to neglected tropical diseases in Brazil. Parasite epidemiology and control,1(2), 56-65. https://doi.org/10.1016/j.parepi.2016.03.002

Mendes, K. D. S, Silveira, R. C. C. P., \& Galvão, C. M. (2008). Revisão integrativa: método de pesquisa para a incorporação de evidências na saúde e na enfermagem. Texto Contexto Enferm, 17(4), 758-64. https://www.scielo.br/pdf/tce/v17n4/18.pdf

Morais, R. C. S. (2015). Aplicabilidade da técnica de PCR em tempo real para caracterização de espécies de Leishmania. [Dissertação de Mestrado em Biociências e Biotecnologia em Saúde, Centro de Pesquisas Aggeu Magalhães, Fundação Oswaldo Cruz]. https://www.arca.fiocruz.br/handle/icict/12756

Navarro, A., Rubiano, L., Arango, J. D., Rojas, C. A., Alexander, N., Saravia, N. G., \& Aronoff-Spencer, E. (2018). Developing mobile health applications for neglected tropical disease research. PLoS Negl Trop Dis, 12(11), e0006791. https://doi.org/10.1371/journal.pntd.0006791

Oliveira, A. R. F., \& Alencar, M. S. M. (2017). O uso de aplicativos de saúde para dispositivos móveis como fontes de informação e educação em saúde. RDBCI: Revista Digital de Biblioteconomia e Ciência da Informação, 15(1), 234-245. https://doi.org/10.20396/rdbci.v15i1.8648137

Opas/Oms. (2020). Informa de Leishmanioses n ${ }^{\circ}$ 9. dezembro, 2020. Informe epidemiológico das Américas. https://iris.paho.org/handle/10665.2/53091

Opas/Oms. (2019). Informe de Leishmanioses $\mathrm{n}^{\mathrm{o}}$ 8. dezembro, 2019. Informe epidemiológico das Américas. https://iris.paho.org/bitstream/há ndle/10665.2/51738/ leishreport8_por.pdf?sequence=1\&isAllowed=y\#: :text=Das\%20mortes\%20por\%20LC\%2FLM,e\%20pa\%C3\%ADs\%2C\%20a m\%C $3 \%$ A9ricas $\% 2 \mathrm{C} \% 202018$.

Pace, D. (2014). Leishmaniasis. Journal Of Infection, 69(1), 10-18. https://doi.org/10.1016/j.jinf.2014.07.016

Pessoa, S. B., \& Martins, A. V. (1982). Parasitologia médica. (11a ed.), Editora Guanabara Koogan.

Rocha, T. A. H., Fachini, L. A., Thumé, E., Silva, N. C., Barbosa, A. C. Q., Carmo, M., \& Rodrigues, J. M. (2016). Saúde Móvel: novas perspectivas para a oferta de serviços em saúde. Epidemiol. Serv. Saúde, Brasília, 25(1), 159-170. https://doi.org/10.5123/S1679-49742016000100016

Rubiano, L., Alexander, N.D. E., Castillo, R. M., Martínez, A. J., Luna, J. A. G., Arango, J. D., Vargas, L., Madriñán, P., Hurtado, L. R., Orobio, Y., Rojas, C. A., Corral, H., Navarro, A., Saravia, N. G., \& Aronoff-Spencer, E. (2021). Adaptation and performance of a mobile application for early detection of cutaneous leishmaniasis. PLoS Negl Trop Dis, 15(2), e0008989. https://doi.org/10.1371/journal.pntd.0008989

Schubach, A. O.; \& Conceição-Silva, F. (2014). Estado da Arte no Tratamento da Leishmaniose Tegumentar Americana no Brasil. Leishmanioses no continente americano. Editora Fiocruz.

Silva, P. E. F., Fonseca-Junior, G. S., Ambrozio, R. B., Costa, M. G. S. T., Machado, G. B., Carvalho, S. F. G., Oliveira, E. J., Jorge, D. C., \& Teix eira, L. A. S. (2020). LeishCare®: A Software Designed for the Management of Individuals with Leishmaniases. Am. J. Trop. Med. Hyg., 103(2), 909-916. 10.4269/ajtmh.19-0178

Souza, C. S. A. (2018). Análise temporal, espacial e fatores associados à mortalidade por leishmaniose tegumentar no Brasil. [Dissertação de Mestrado em Saúde Coletiva, Instituto René Rachou, Fundação Oswaldo Cruz). https://www.arca.fiocruz.br/handle/icict/34167

Taheri, A. R., Alikhani, S., Sazgarnia, R., Salehi, M., \& Amlashi, S. V. (2017). Digital volumetric measurement of cutaneous leishmaniasis lesions: Blur estimation method. Indian Journal of Dermatology, Venereology, and Leprology, 83(3), 307-311. https://doi.org/10.4103/ijdvl.IJDVL_134_16

Who. World Health Organization. (2020). Ending the neglect to attain the Sustainable Development Goals: a road map for neglected tropical diseases 20212030. World Health Organization.

Who. World Health Organization. (2017). https://www.who.int/news/item/19-04-2017-unprecedented-progress-against-neglected-tropical-diseases-whoreports

Who. World Health Organization. Control of the leishmaniases: report of a meeting of the WHO Expert Committee on the Control of Leishmaniases. World Health Organization, 2010. ISBN 9 